\title{
Mixing of spin and orbital angular momenta via second-harmonic generation in plasmonic and dielectric chiral nanostructures
}

\author{
Xiaoyan Y. Z. Xiong, ${ }^{1}$ Ahmed Al-Jarro, ${ }^{2}$ Li Jun Jiang, ${ }^{1,}{ }^{*}$ Nicolae C. Panoiu, ${ }^{2}$ and Wei E. I. Sha ${ }^{1}$ \\ ${ }^{1}$ Department of Electrical and Electronic Engineering, University of Hong Kong, Hong Kong \\ ${ }^{2}$ Department of Electronic and Electrical Engineering, University College London, Torrington Place, London WC1E 7JE, United Kingdom
}

(Received 8 January 2017; revised manuscript received 2 April 2017; published 19 April 2017)

\begin{abstract}
We present a theoretical study of the characteristics of the nonlinear spin-orbital angular momentum coupling induced by second-harmonic generation in plasmonic and dielectric nanostructures made of centrosymmetric materials. In particular, the connection between the phase singularities and polarization helicities in the longitudinal components of the fundamental and second-harmonic optical fields and the scatterer symmetry properties are discussed. By in-depth comparison between the interaction of structured optical beams with plasmonic and dielectric nanostructures, we have found that all-dielectric and plasmonic nanostructures that exhibit magnetic and electric resonances have comparable second-harmonic conversion efficiency. In addition, mechanisms for second-harmonic enhancement for single and chiral clusters of scatterers are unveiled and the relationships between the content of optical angular momentum of the incident optical beams and the enhancement of nonlinear light scattering is discussed. In particular, we formulate a general angular momenta conservation law for the nonlinear spin-orbital angular momentum interaction, which includes the quasi-angular-momentum of chiral structures with different-order rotational symmetry. As a key conclusion of our study relevant to nanophotonics, we argue that all-dielectric nanostructures provide a more suitable platform to investigate experimentally the nonlinear interaction between spin and orbital angular momenta, as compared to plasmonic ones, chiefly due to their narrower resonance peaks, lower intrinsic losses, and higher sustainable optical power.
\end{abstract}

DOI: 10.1103/PhysRevB.95.165432

\section{INTRODUCTION}

Optical vortex beams carrying orbital and spin angular momentum have attracted great interest because of their important technological applications, including to optical tweezers [1-3], high-capacity optical communications [4,5], and quantum key distribution [6]. They have also been explored in various nonlinear optical processes recently, e.g., orbital angular momentum (OAM) entanglement between photons created by spontaneous parametric down conversion [7], OAM conservation in nonlinear wave mixing [8], and enhanced nonlinear optical activity and nonlinear phase control $[9,10]$. In this context, the process of the second-harmonic ( $\mathrm{SH}$ ) generation (SHG), where two photons with fundamental frequency (FF), $\omega$, convert into one photon with $\mathrm{SH}$ frequency, $\Omega=2 \omega$, is the nonlinear process most used in studies of OAM, chiefly due to the relative simplicity of its experimental implementation. Most previous studies in this area focused on the manipulation and control of OAM by isotropic or chiral bulk nonlinear optical media during the SH conversion process [11-15].

With recent advances in nanotechnology, it has become possible to investigate the SHG from plasmonic and alldielectric nanoparticles, which in most cases of practical interest are made of centrosymmetric optical materials [16-21]. The SHG technique has established itself as a powerful and promising tool for surface characterization, biomedicine, and nanotechnology applications [22-25]. Despite this, a complete understanding of the spin-orbit interaction (SOI) pertaining to the surface SHG in nanostructures is still missing.

\footnotetext{
*1jiang@eee.hku.hk
}

Plasmonic nanostructures that support localized surface plasmon-polaritons (SPPs) find pervasive use in nonlinear optical devices, chiefly because plasmon-enhanced optical near-fields allow nonlinear optical processes that ordinarily are weak to be significantly enhanced [26]. On the other hand, large ohmic losses in plasmonic materials limit the $\mathrm{SH}$ conversion efficiency and optical power at which nonlinear plasmonic devices can be operated. Alternatively, dielectric nanostructures exhibit a much lower intrinsic loss and hence a much higher power ablation threshold, the drawback being that the local field enhancement is smaller than in the case of plasmonic structures. Whereas a definitive conclusion has not been reached yet, preliminary studies suggest that dielectric nanostructures might provide higher frequency conversion efficiency as compared to their plasmonic counterparts, especially if resonant modes are employed [27,28]. In this connection, it should be stressed that investigations aiming to assess the relative benefits provided by nonlinear plasmonic and dielectric nanostructures are particularly helpful in clarifying this matter.

In this work, SOI in plasmonic and dielectric nanostructures upon the SHG process is studied by using LaguerreGaussian (LG) beams [29]. These are the ubiquitous laboratory implementations of optical vortices [30,31], their physical properties making them particularly suitable to study the angular momentum of optical fields. In particular, LG beams in the $\mathrm{LG}_{p l}$ configuration have a concentric-ring-like structure with $p$ radial nodes in the intensity profile and a characteristic azimuthal phase variation of $e^{i l \phi}$, where $\phi$ is the azimuthal coordinate and $l$ is the topological charge of the beam measuring the phase variation, modulo $2 \pi$, along a closed curve containing the center of the vortex ( $l$ is also called OAM photon number). Generally, optical beams can also contain spin angular momentum (SAM) associated with their polarization states, $\sigma$. Thus, $\sigma=0$ for linearly polarized 


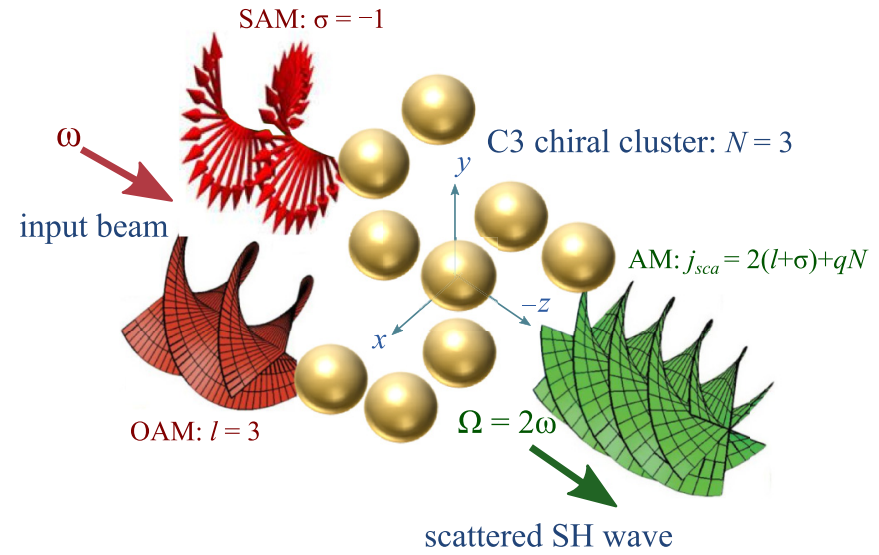

FIG. 1. Schematic of the nonlinear spin-orbital angular momentum (AM) coupling induced by second-harmonic generation (SHG) in a chiral cluster with $N$-fold rotation symmetry. Both the frequency and angular momentum have doubled during the SHG process. In addition, the quasi-angular-momentum, defined as the order of the rotational symmetry of the chiral cluster $(N)$, can be transferred to the scattered SH wave to conserve the total angular momentum. $q$ is an integer and $q=0, \pm 1, \pm 2, \pm 3, \ldots$.

light and $\sigma=1(\sigma=-1)$ for left (right) circularly polarized (LCP, RCP) light. In this article, we provide a comprehensive description of the scattering of circularly polarized LG beams from single plasmonic and dielectric nanospheres made of centrosymmetric materials, chiral clusters made of such nanospheres and with specific rotational symmetry properties, and chiral nanostructures with symmetry properties identical to those of the nanosphere clusters. More specifically, we investigate the characteristics of the SOI mediated by the considered nanostructures and its relation to the properties of the linear and nonlinear (SHG) scattering processes.

The paper is organized as follows. In the next section we present the main results pertaining to the linear and nonlinear light scattering from single nanoparticles as well as chiral clusters of nanoparticles and chiral nanostructures. The SOI phenomena in these structures are analyzed by exploring the properties of the near- and far-field. A general selection rule including the quasi-angular-momentum matching for the chiral structures is also formulated. Furthermore, we compare the SH conversion efficiency of chiral structures made of plasmonic and dielectric materials. Finally, in the last section, we summarize the main conclusions of our study.

\section{RESULTS AND DISCUSSION}

We consider a chiral cluster of nanospheres with $N$-fold rotation symmetry, which is surrounded by air and irradiated by a circularly polarized LG beam carrying spin and orbital angular momentum, as shown in Fig. 1. We calculate numerically the light scattering at the fundamental frequency (FF) and SH from plasmonic and dielectric nanostructures using the boundary element method [32]. Only the surface contribution to the SHG is considered for centrosymmetric materials [33-35]. The linear and nonlinear fields and currents at the surface of the nanostructure are used to characterize the SHG.

In this work we consider generic plasmonic and dielectric centrosymmetric materials, namely gold $(\mathrm{Au})$ and silicon $(\mathrm{Si})$.
According to a model that is widely used in the study of the SHG in centrosymmetric media [36], the surface nonlinear polarization is described by a surface nonlinear susceptibility and can be written as

$$
\mathbf{P}_{s}(\mathbf{r}, \Omega)=\epsilon_{0} \hat{\chi}_{s}^{(2)}: \mathbf{E}(\mathbf{r}, \omega) \mathbf{E}(\mathbf{r}, \omega) \delta\left(\mathbf{r}-\mathbf{r}_{s}\right),
$$

where $\mathbf{r}_{s}$ defines the surface, $\hat{\chi}_{s}^{(2)}$ is the surface second-order susceptibility tensor, $\mathbf{E}(\mathbf{r}, \omega)$ is the fundamental field taken inside the material, and the Dirac function defines the surface characteristic of the nonlinear polarization.

Except for the case when the surface contains structural features with intrinsic chirality, the surface of centrosymmetric media possesses an isotropic mirror-symmetry plane perpendicular to the interface. Then, the surface nonlinear susceptibility $\hat{\chi}_{s}^{(2)}$ has only three independent components, i.e., $\hat{\chi}_{s, \perp \perp \perp}^{(2)}, \hat{\chi}_{s, \perp\|\|,}^{(2)}$, and $\hat{\chi}_{s,\|\perp\|}^{(2)}=\hat{\chi}_{s,\|\| \perp \perp}^{(2)}$, where the symbols $\perp$ and $\|$ refer to the directions normal and tangent to the surface, respectively. The susceptibility components of $\mathrm{Au}$ are $\hat{\chi}_{s, \perp \perp \perp}^{(2)}=1.59 \times 10^{-18} \mathrm{~m}^{2} / V, \hat{\chi}_{s,\|\| \perp}^{(2)}=$ $\hat{\chi}_{s,\|\perp\|}^{(2)}=4.63 \times 10^{-20} \mathrm{~m}^{2} / V$, and $\hat{\chi}_{s, \perp\|\|}^{(2)}=0$ [37], while for Si they are $\hat{\chi}_{s, \perp \perp \perp}^{(2)}=6.5 \times 10^{-18} \mathrm{~m}^{2} / V, \hat{\chi}_{s,\|\| \perp}^{(2)}=\hat{\chi}_{s,\|\perp\|}^{(2)}=$ $3.5 \times 10^{-19} \mathrm{~m}^{2} / V$, and $\hat{\chi}_{s, \perp\|\|}^{(2)}=1.3 \times 10^{-19} \mathrm{~m}^{2} / V$ [38].

\section{A. A single nanosphere}

We begin our study with an analysis of the scattering of polarized LG beams from a single gold nanosphere, as the nanosphere possesses well-defined multipole-type optical modes. The gold nanosphere placed at the system origin and embedded in vacuum is illuminated by a LG beam propagating along the $-z$ axis with its minimum waist in the $x y$ plane. The LG beam carries a topological charge, $l$, and it has no nodes along the radial coordinate (however, it can be zero at $r=0$ ); i.e., we consider $\mathrm{LG}_{0 l}$ beams. In addition, we assume that the beam is circularly polarized, which means it also carries SAM, i.e., $\sigma=+1$ for LCP and $\sigma=-1$ for RCP. The total angular momentum carried by the circularly polarized LG beam is characterized by the integer $j=l+\sigma$. In our analysis, the dielectric constant for gold is taken from experimental data [39].

The fundamental and SH scattering cross sections, as a function of the radius of the gold nanosphere and the wavelength at the FF, $\lambda_{\mathrm{FF}}$, are shown in Fig. 2 . Due to the excitation of a surface plasmon resonance at the incident wavelength $\lambda_{\mathrm{FF}}=520 \mathrm{~nm}$, the gold nanosphere has the largest scattering cross section for both LCP and RCP LG beams with the topological charge of $l=1$. The resonant responses are marked with dashed lines in Figs. 2(a) and 2(b). The SH scattering cross-section maps exhibit a resonant response exactly at $\lambda_{\mathrm{SH}}=260 \mathrm{~nm}$ because of the plasmon-induced optical near-field enhancement.

Although the resonances occur at the same frequency for LG beams with different polarization, the amplitude and phase of the near-field fundamental and SH longitudinal-field components $\left(E_{z}\right)$ have different features due to the SOI. Figures 3(a)-3(d) and Figs. 3(e)-3(h) display the magnitude and phase of longitudinal fundamental and $\mathrm{SH}$ fields, respectively, calculated in a section parallel to the $x y$ plane at the resonant wavelength $\lambda_{\mathrm{FF}}=520 \mathrm{~nm}\left(\lambda_{\mathrm{SH}}=260 \mathrm{~nm}\right)$. The 

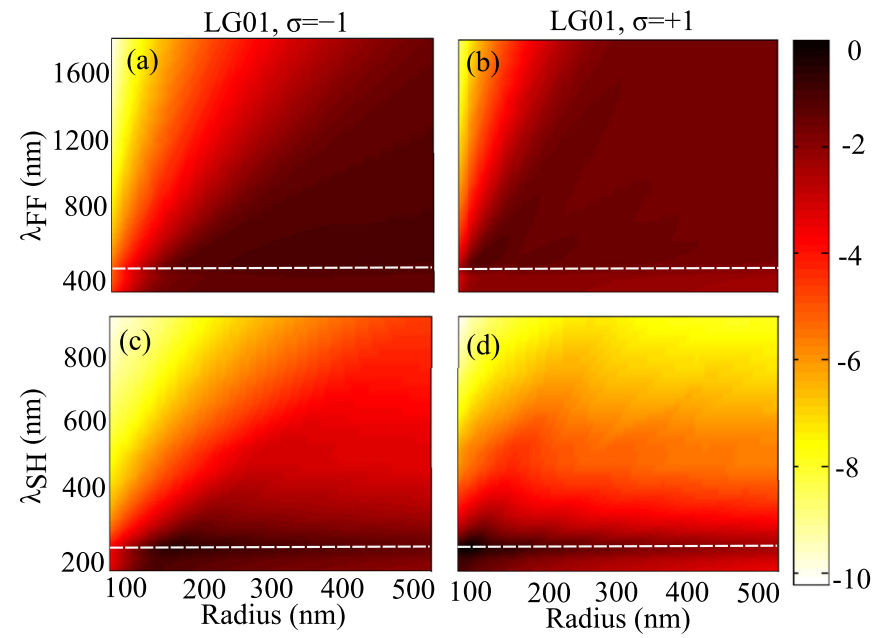

FIG. 2. Spectra of the normalized fundamental (top panels) and $\mathrm{SH}$ (bottom panels) scattering cross section vs radius of the gold sphere illuminated by LCP $(\sigma=+1)$ and $\operatorname{RCP}(\sigma=-1)$ LG beams with the OAM of $l=1$. The LG beam waist is $w_{0}=2 \lambda_{\mathrm{FF}}$. Dashed lines correspond to the resonant responses in the bulk. Panels are plotted on a logarithmic scale.

radius of the gold sphere is fixed to $100 \mathrm{~nm}$ and the observation plane is located $40 \mathrm{~nm}$ away from the backside of the sphere (the side farthest from the source).

It can be seen that for the LCP light $(\sigma=+1)$ the amplitude of the scattered fundamental and SH fields vanishes at the axis of the beam due to the phase singularities at this point [see Figs. 3(a) and 3(e)]. Moreover, the phase profile of the SH field [Fig. 3(f)] has twice as many nodes along a closed path that contains the origin as compared to the profile of the fundamental field [Fig. 3(b)]. This means that during the photon conversion process not only has the frequency doubled, but also the photon angular momentum has doubled its value. In other words, both the energy and the total angular momentum are conserved in the nonlinear interaction of light with the nanosphere. In contrast, in the case of the RCP $\mathrm{LG}_{0 l}$ beam, the amplitudes of the forward scattered fundamental [Fig. 3(c)] and SH fields [Fig. 3(g)] are no longer zero at the beam axis due to the linear and nonlinear SOI upon resonant light scattering from the $\mathrm{Au}$ nanosphere. That is, the phase vorticity related to OAM $(l=1)$ cancels the polarization helicity related to $\operatorname{SAM}(\sigma=-1)$ in the incident beam, producing at both the FF and $\mathrm{SH}$ a field whose phase is independent of the azimuthal angle [Figs. 3(d) and 3(h)] without phase singularities at the beam axis. This means that the value of $j=l+\sigma$ is preserved during the light interaction with the nanosphere; i.e., the total angular momentum is conserved. This is not surprising as the sphere has $\mathcal{C}_{\infty}$ symmetry with respect to rotations around the beam axis and consequently angular momentum cannot be transferred between the beam and nanosphere.

In order to understand the specific differences between the characteristics of scattering of LG beams from metallic and dielectric nanoparticles, we consider next the scattering of such beams from silicon nanospheres. The linear scattering process has been discussed in [29], but for the sake of completeness we also summarize here the main ideas. The radius of the nanosphere is chosen so that the wavelengths of the optical resonances are located in the spectral regions where the intrinsic optical losses of silicon can be neglected, i.e., $\lambda \gtrsim$ $1.2 \mu \mathrm{m}$, and the wavelength dependence of the permittivity of silicon is described via the Sellmeier equation [40]. The incident LG beams carry an OAM of $l=1,4$ and SAM of either $\sigma=+1$ or $\sigma=-1$. Figure 4 shows the fundamental and $\mathrm{SH}$ scattering cross section maps as a function of the nanosphere radius and wavelength. Different from the broadspectrum optical response from a single gold sphere (see Fig. 2), the spectra of the silicon nanosphere are much richer in spectral features, with a series of resonant bands, which become narrower and more closely spaced as the wavelength decreases. The richer spectra of the silicon nanosphere are
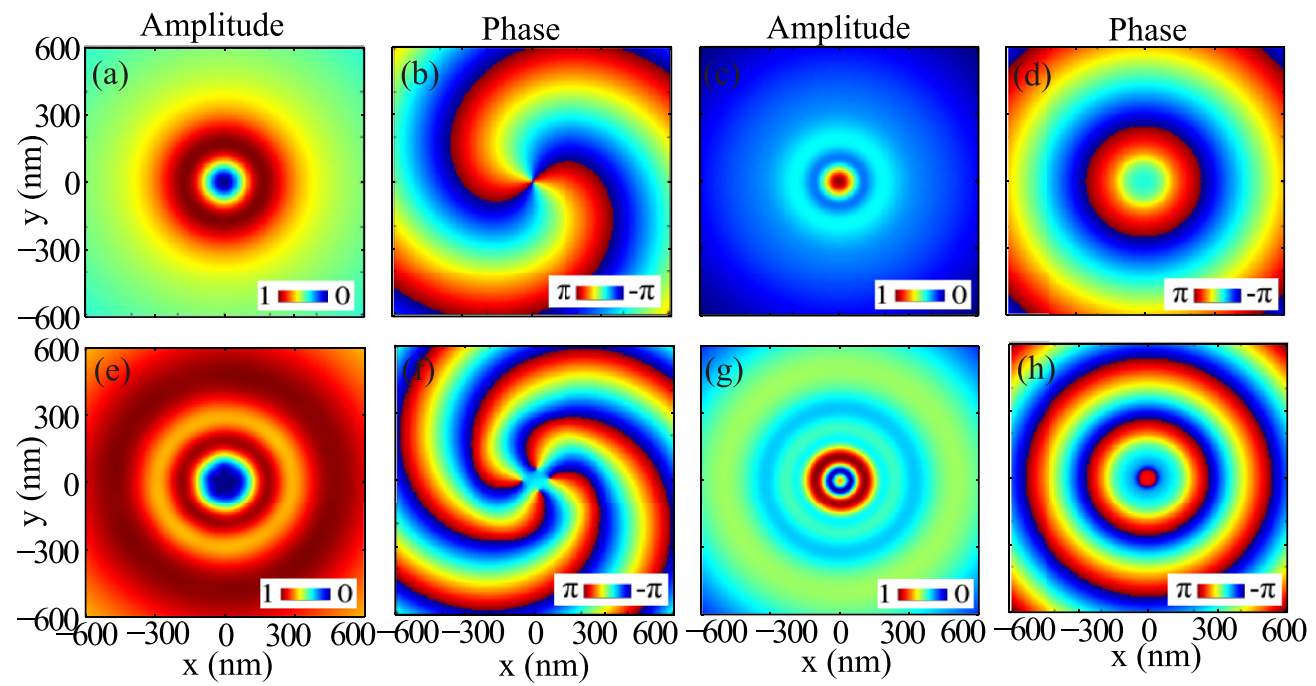

FIG. 3. Amplitudes and phases of the near-field longitudinal-field component $\left(E_{z}\right)$ of the fundamental (top panels) and SH field (bottom panels) scattered from a gold sphere illuminated by the LCP $(\sigma=+1)$ and RCP $(\sigma=-1)$ LG beams with the OAM of $l=1$. The LG beam waist is $w_{0}=2 \lambda_{\mathrm{FF}}$ and the wavelength of the beam is $\lambda_{\mathrm{FF}}=520 \mathrm{~nm}$. Radius of the gold sphere is $100 \mathrm{~nm}$. Panels (a), (b), (e), and (f) correspond to LCP beam $(\sigma=+1)$, whereas panels (c), (d), (g), and (h) to RCP beam $(\sigma=-1)$. 

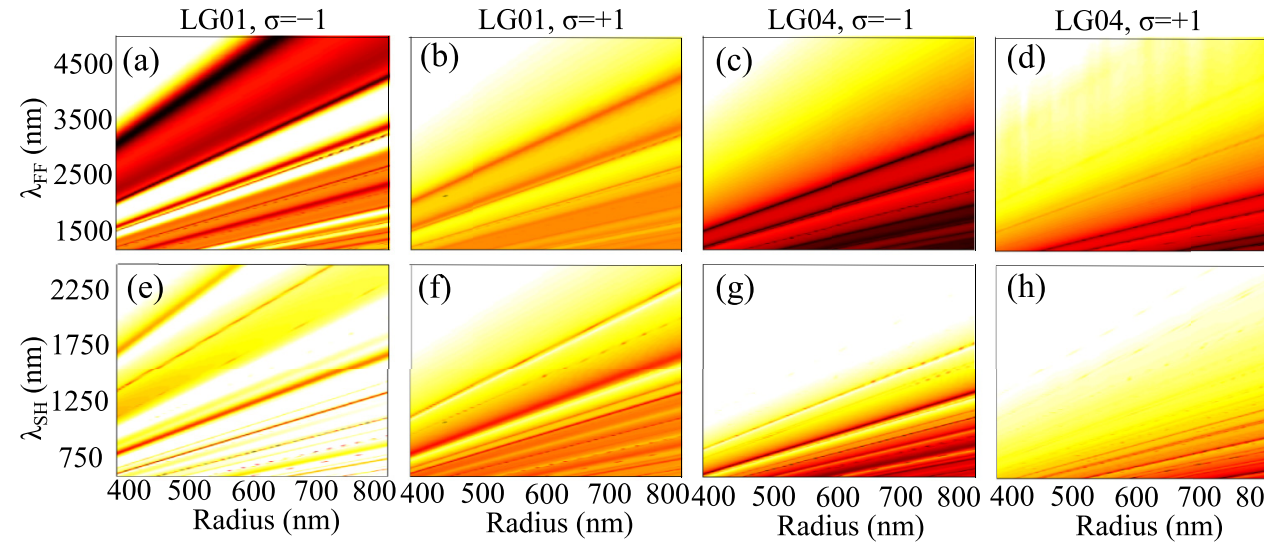

(h)

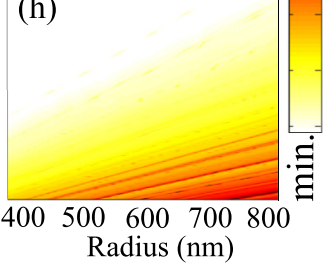

FIG. 4. Spectra of the normalized fundamental (top panels) and SH (bottom panels) scattering cross section vs radius of the silicon sphere illuminated by LCP $(\sigma=+1)$ and RCP $(\sigma=-1) \mathrm{LG}$ beams with OAM of $l=1$ and $l=4$. The waist of the LG beam is $w_{0}=2 \lambda_{\mathrm{FF}}$. Figures are plotted on a logarithmic scale.

due to two effects: One is the size effect; that is, the larger silicon nanosphere supports more resonances. The other is the excitation of magnetic modes in the silicon nanosphere as will be illustrated later. Equally important, with the increase of the total angular momentum $j=l+\sigma$, more resonant bands are suppressed in the long-wavelength region for both the fundamental [Figs. 4(a)-4(d)] and SH [Figs. 4(e)-4(h)] fields. As will be explained later, this phenomenon is closely related to the amount of angular momentum carried by the LG beams and that contained in the optical (Mie) modes of the silicon nanosphere.

The physical origin of these resonance bands can be understood by considering a simpler scattering configuration, i.e., a linearly polarized plane wave $(\sigma=0)$. Thus, we calculate the scattering spectrum of a single silicon nanosphere with the radius of $500 \mathrm{~nm}$ excited by a linearly polarized plane wave. Figure 5(a) illustrates the decomposition of the scattering cross section in terms of transverse magnetic (TM) and transverse electric (TE) multipoles, as the silicon nanosphere supports both electric and magnetic optical modes. When comparing the scattering spectra corresponding to illumination with plane wave and LG beams with $l=1$ and $l=4$, as displayed in Fig. 5(b), we found that the resonance peaks of the LG beams are located at the same positions as those of the plane wave excitation. More specifically, the scattering spectrum of the RCP LG beam with $l=1$ and $\sigma=-1$ is almost identical to that corresponding to the linearly polarized plane wave. This is because the incident field profile of the LG beam is similar to that of the plane wave, and because the latter is achiral, considering that the polarization helicity and phase vorticity cancel each other and the beam waist $w_{0}=2 \lambda_{\mathrm{FF}}$ is much larger than the radius of the silicon nanosphere.

Because of the conservation of the projection along the beam axis of the total angular momentum, the $\mathrm{LG}_{0 l}$ beam with $\sigma= \pm 1$ can only excite multipole modes of the nanosphere with $n \geqslant l+\sigma$ ( $n$ is the angular momentum quantum number of spherical harmonics $Y_{n m}, m$ is the magnetic quantum number, $-n \leqslant m \leqslant n$ and $n \geqslant 1$ ), and therefore all multipole modes with radial quantum number smaller than $l+\sigma$ will be completely suppressed. This is illustrated in Fig. 5(b), where the $\mathrm{LG}_{01}$ beam with $\sigma=+1$ only excites modes with $n \geqslant 2$, i.e., the $\mathrm{TE}_{1}$ and $\mathrm{TM}_{1}$ resonances vanish, whereas the $\mathrm{LG}_{04}$ beam with $\sigma=-1$ only excites modes with $n \geqslant 3$, the scattering channels of $\mathrm{TE}_{1}, \mathrm{TM}_{1}, \mathrm{TE}_{2}$, and $\mathrm{TM}_{2}$ being completely suppressed. Similarly, the $\mathrm{LG}_{04}$ beam with $\sigma=+1$ only excites optical modes with $n \geqslant 5$.

Figure 5(c) presents the $\mathrm{SH}$ scattering cross section spectra. When comparing Figs. 5(b) and 5(c), we can observe that both the fundamental and $\mathrm{SH}$ spectra contain resonances at several specific wavelengths of the incident beam, e.g., at $1691 \mathrm{~nm}$, $2100 \mathrm{~nm}$, and $2865 \mathrm{~nm}$. The strong SH radiation at these
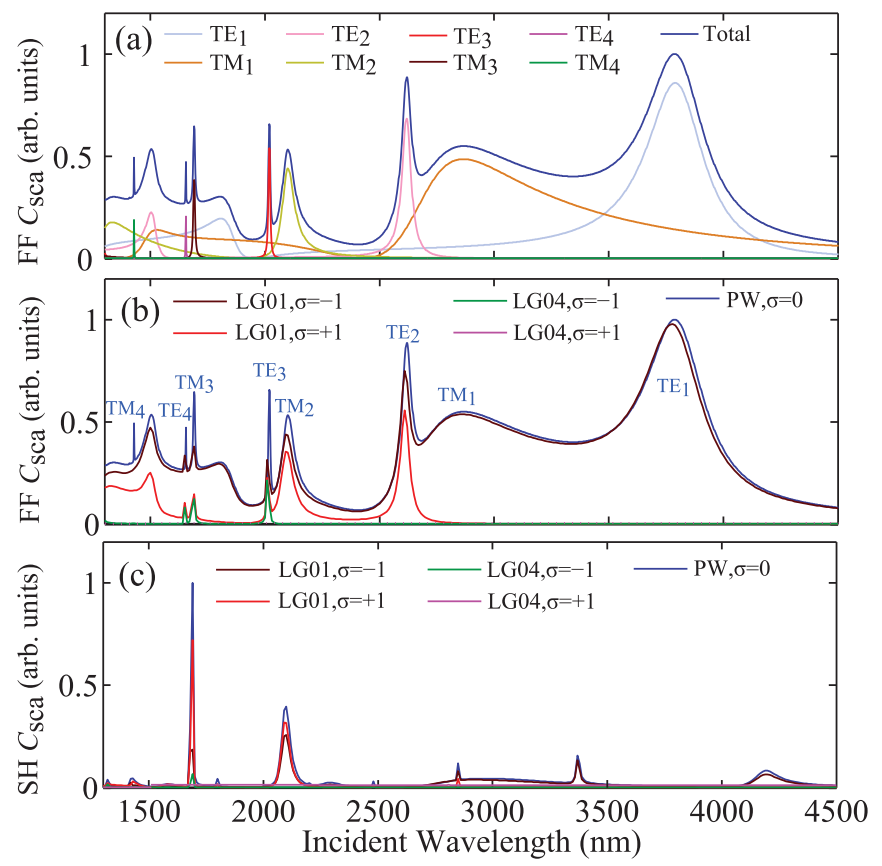

FIG. 5. Normalized scattering cross section, $C_{\text {sca }}$, spectra of a silicon nanosphere with radius of $500 \mathrm{~nm}$. The incoming excitation is a linearly polarized plane wave or circularly polarized LG beams. (a) Fundamental $C_{\text {sca }}$ and its multipole expansion for linearly polarized plane wave excitation. (b) Fundamental $C_{\text {sca }}$ for linearly polarized plane wave and circularly polarized LG beam excitations. (c) $\mathrm{SH} C_{\mathrm{sca}}$ for linearly polarized plane wave and circularly polarized LG beam excitations. 

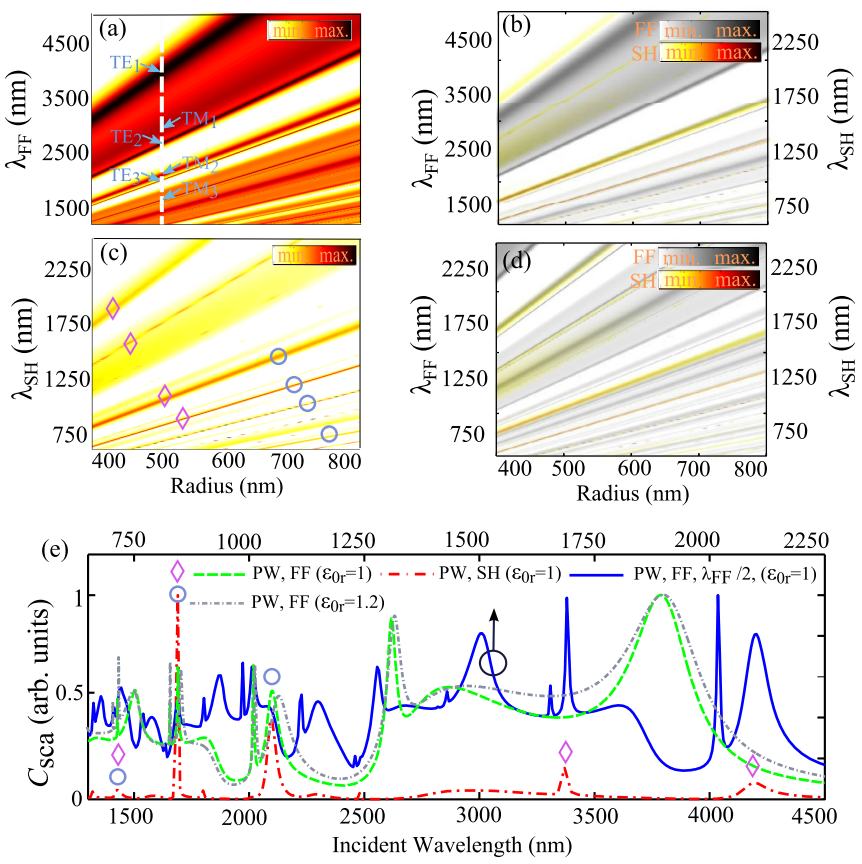

FIG. 6. Spectra of the normalized scattering cross section vs radius of the silicon sphere illuminated by linearly polarized plane waves. (a) Fundamental field and (c) SH field with incident wavelength ranging from $1300 \mathrm{~nm}$ to $4800 \mathrm{~nm}$; (b) overlap between maps (a) and (c); (d) overlap between map (c) and the map at the FF $\left(\lambda_{\mathrm{FF}}\right.$ ranges from $650 \mathrm{~nm}$ to $2400 \mathrm{~nm}$ ); (e) fundamental and $\mathrm{SH}$ scattering cross section of the silicon sphere with radius fixed to $500 \mathrm{~nm}$ surrounded by background material with different relative permittivity $\epsilon_{0 r}\left(\epsilon_{0 r}=1\right.$ and $\left.\epsilon_{0 r}=1.2\right)$. In (b) and (d), the left $\left(\lambda_{\mathrm{FF}}\right)$ and right $\left(\lambda_{\mathrm{SH}}\right)$ axes are used for the fundamental and SH scattering cross section, respectively. Panels (a)-(d) are plotted in a logarithmic scale. Panel (e) is plotted in a linear scale.

wavelengths is due to the resonant enhancement of the optical field at the FF; that is, when fundamental field resonance occurs at these wavelengths, the local field is enhanced and thus more SH light is generated. However, we can also find in the $\mathrm{SH}$ spectra resonances that have no counterpart in the linear spectra, e.g., those at $\lambda_{\mathrm{FF}}=3382 \mathrm{~nm}$ and $\lambda_{\mathrm{FF}}=4200 \mathrm{~nm}$. These peaks are due to the fact that optical modes exist at half the wavelength of the excitation beam. For example, when the fundamental wavelength is $3382 \mathrm{~nm}$, SH currents are generated at $1691 \mathrm{~nm}$. These SH sources resonantly excite the optical mode of the sphere that exists at this frequency and, consequently, the scattered SH field is significantly enhanced.

In order to further clarify the origin of these two kinds of SH enhancement mechanisms, we compare in Figs. 6(a) and 6(c) the maps of the fundamental and SH scattering cross sections corresponding to linearly polarized plane wave excitation $(\sigma=0)$, the incident wavelength ranging from $1300 \mathrm{~nm}$ to $4800 \mathrm{~nm}$. Figure 6(b) shows the overlap between the map at the FF [ $\lambda_{\mathrm{FF}}$ ranges from $1300 \mathrm{~nm}$ to $4800 \mathrm{~nm}$; Fig. 6(a)] and SH map $\left[\lambda_{\text {SH }}\right.$ ranges from $650 \mathrm{~nm}$ to $2400 \mathrm{~nm}$; Fig. 6(c)]. The overlapping resonance bands, denoted by circles in Fig. 6(c), demonstrate the FF-resonance enhancement mechanism. Moreover, we also calculate the map of the scattering cross section at the FF with the excitation wavelength ranging from $650 \mathrm{~nm}$ to $2400 \mathrm{~nm}$. The overlap between this map and the map of the scattering cross section at $\mathrm{SH}$, with $\lambda_{\mathrm{SH}}$ ranging from $650 \mathrm{~nm}$ to $2400 \mathrm{~nm}$, is shown in Fig. 6(d). The overlapping bands correspond in this case to the $\mathrm{SH}$-resonance enhancement mechanism and are denoted by diamonds in Fig. 6(c). Interestingly enough, there are two double-resonance bands exhibiting both the FF-resonance and SH-resonance enhancement mechanisms, the corresponding intensity of the generated SH being particularly large when this double-resonance condition is satisfied.

\section{B. Chiral nanostructures with rotational symmetry}

Chiral nanostructures have been widely investigated for generating or interacting with OAM beams [41-43]. Armed with the knowledge pertaining to the linear and nonlinear light scattering from a single nanosphere, we consider now the scattering of LG beams from planar chiral nanostructures [44] with $N$-fold rotational symmetry. For the sake of specificity, we set $N=3$ in our calculations. To begin with, we consider a chiral nanostructure consisting of 10 identical nanospheres arranged in an Archimedes-like spiral configuration, as schematically shown in the inset of Fig. 7(b), and denoted as C3-D. The second chiral nanostructure is the "continuous" counterpart of the first one and is denoted as $\mathrm{C} 3-\mathrm{C}$ in the inset of Fig. 7(b). The chiral nanostructures lie in the $x y$ plane in such a way that their center coincides with the origin of the coordinate system. The LCP and RCP LG beams with the OAM of $l=4$, $\mathrm{LG}_{04}$, are assumed to propagate along the $-z$ direction with the beam axis passing through the origin. Moreover, we assume that in both cases the chiral nanostructures are made of silicon
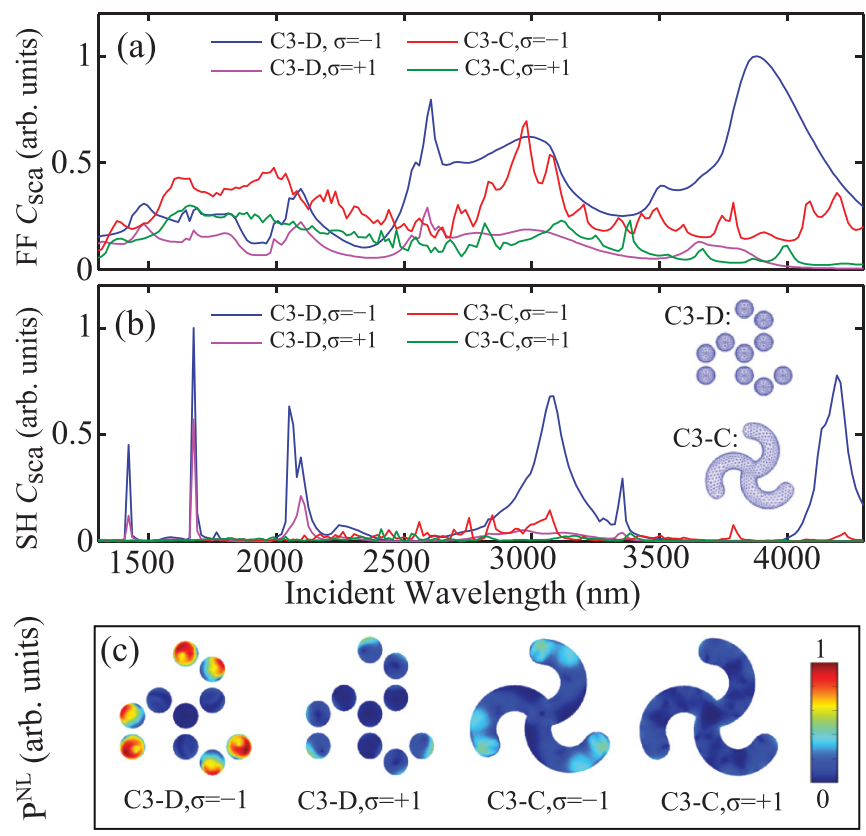

FIG. 7. Spectra of the normalized scattering cross section, $C_{\text {sca }}$, of dielectric chiral nanostructures illuminated by $\operatorname{LCP}(\sigma=+1)$ and $\operatorname{RCP}(\sigma=-1)$ LG beams with the OAM quantum number of $l=4$. The LG beam waist is $w_{0}=2 \lambda_{\mathrm{FF}}$. (a) $C_{\mathrm{sca}}$ at the FF. (b) $C_{\mathrm{sca}}$ at SH. (c) Spatial distributions of the nonlinear polarization currents at the surface. 
because in this case the spectra of the scattering cross section have a larger set of features. For a more reliable comparison, the radius of the spheres in the cluster is assumed to be equal to the radius of the cross section of the arms of the continuous C3-C nanostructure and set to $500 \mathrm{~nm}$.

The scattering spectra at the FF were calculated and are depicted in Fig. 7(a). Despite the "noisy" aspect of spectra in Fig. 7, important features can be deduced. It shows that the scattering characteristics of the chiral structures strongly depend on the polarization state of the LG beam. Thus, for the $\mathrm{LG}_{04}$ beam with the $\operatorname{RCP}(\sigma=-1)$, the quantum number of the total angular momentum of the incident beam is $j=l+\sigma=3$, which matches the rotational symmetry order of the two chiral structures, $N=3$. This quasi-angularmomentum matching between the incident beam and the chiral scatterers greatly increases the light scattering at the FF. Although the C3-C nanostructure illuminated by the RCP LG $\mathrm{L}_{04}$ beam shows strong light scattering in the linear regime, this is not the case for its $\mathrm{SH}$ radiation, which is presented in Fig. 7(b). Comparing the optical response of the two structures, one can see that the C3-D nanostructure has larger radiation intensity for both the fundamental and SH fields.

These results clearly prove the critical role played by the resonances of single nanospheres in the scattering processes at the FF and $\mathrm{SH}$. In particular, the scattering spectra of the C3-D nanostructure have clearly defined and pronounced spectral peaks corresponding to resonances supported by individual nanospheres in the cluster. To better illustrate the $\mathrm{SH}$ enhancement stemming from excitation of nanosphere resonances, we plot in Fig. 7(c), for both chiral nanostructures, the distribution of the surface nonlinear polarization currents. It is evident that the C3-D cluster illuminated by the $\mathrm{LG}_{04}$ beam with $\sigma=-1$ displays the largest nonlinear polarization currents, which in turn give rise to the strongest $\mathrm{SH}$ radiation.

In order to reveal the physical origin of the SH resonant peaks for the chiral nanostructures, we compare the fundamental and SH scattering spectra of the silicon chiral cluster and the fundamental scattering spectra of a single silicon nanosphere, as per Fig. 8. The incident waves are the LG beams and plane waves for the chiral cluster and the signal nanosphere, respectively. At the FF, the spectral locations of the scattering peaks of the chiral cluster almost coincide with those corresponding to a single nanosphere, except for small deviations at long wavelengths caused by the mutual optical coupling between the nanospheres. The SH peaks around $3000 \mathrm{~nm}$ show a broadband enhancement resulting from the mutual coupling, which also can be confirmed by the resonance splitting [Fig. 8(a)] and shift [Fig. 8(b)] marked by dashed boxes in the fundamental spectra of the cluster. On the other hand, high-order whispering gallery modes, which are modes whose OAM is different from zero, supported by a dielectric sphere are more confined and have lower radiation loss at shorter wavelengths, leading to weaker coupling. This suggests that the strong fundamental scattering of the chiral cluster is primarily due to the resonances of individual nanospheres in the cluster. The SH scattering peaks having both FF and $\mathrm{SH}$ resonances enhancements are marked in Fig. 8 by circles and diamonds, respectively. We stress that the resonance peaks located in the long-wavelength region are due to the SH-resonance enhancement mechanism in the cluster,
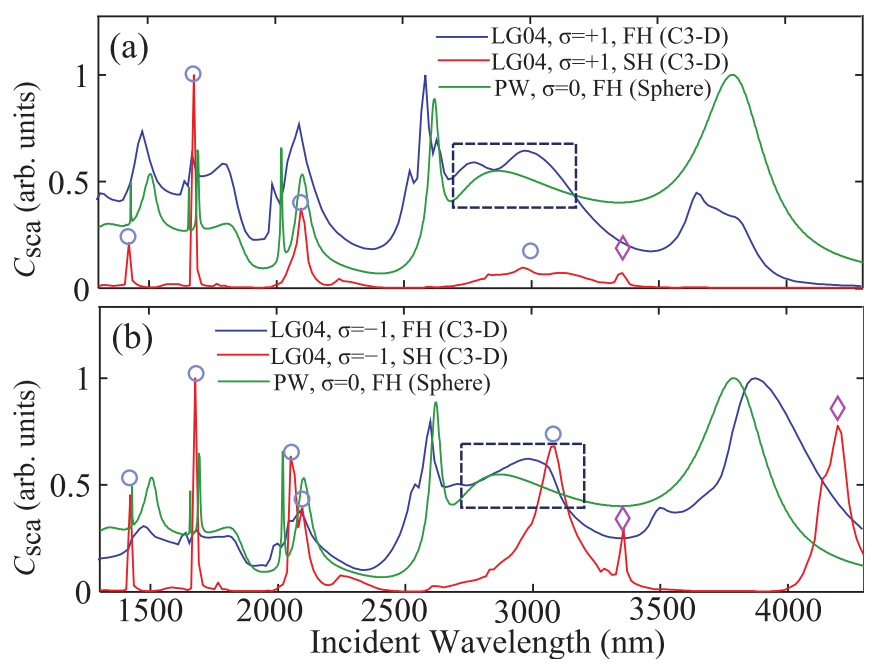

FIG. 8. Comparisons between the fundamental and SH scattering spectra of the silicon chiral cluster with 3-fold rotational symmetry and the fundamental scattering spectra of a single silicon nanosphere. (a) $\mathrm{LG}_{04}, \sigma=+1$. (b) $\mathrm{LG}_{04}, \sigma=-1$.

because the resonance scattering peaks of a single nanosphere are suppressed at long wavelengths, as illustrated in Fig. 5.

For the nanostructures with $N$-fold rotational symmetry, the incident beam with well-defined angular momentum can only scatter into a set of modes with specific values of the total angular momentum. In order to describe how nanostructures with $N$-fold rotational symmetry affect the transfer of angular momentum from the incident field to the scattered field during both linear and nonlinear interaction processes, we formulate a general angular momentum conservation law. It can be written as

$$
j_{\mathrm{sca}}=s(l+\sigma)+q N,
$$

where $l$ and $\sigma$ are the orbital and spin angular momentum numbers of the incident beam, respectively, $s$ denotes the order of harmonic generation ( $s=1$ for linear processes, $s=2$ for $\mathrm{SH}$ generation, etc.), $N$ is the quasi-angular-momentum number possessed by the nanostructure with $N$-fold rotational symmetry, $q$ is an integer $q=0, \pm 1, \pm 2, \pm 3, \ldots$, and $j_{\text {sca }}$ is the total angular momentum number of the scattered field. Equation (2) implies that the total angular momenta of the scattered fundamental or SH fields depend not only on the angular momenta of the incident beam but also on the rotational symmetry properties of the scatterer. The quasi-angular-momentum of the scatterer can be transferred to the scattered wave in order to conserve the total angular momentum. Note that this is the rotational analog of the conservation of momentum upon scattering of electromagnetic waves by diffraction gratings, when the quasimomentum of the grating must be taken into account when describing the light scattering from such optical structures.

To validate this angular momentum conservation law, the total angular momentum of the scattered fundamental and $\mathrm{SH}$ fields from the C3-D chiral cluster is analyzed by the multipole expansion method [45]. Figure 9 shows the relative contributions of the electric and magnetic multipoles to the total angular momentum of the scattered fields. For the incident 

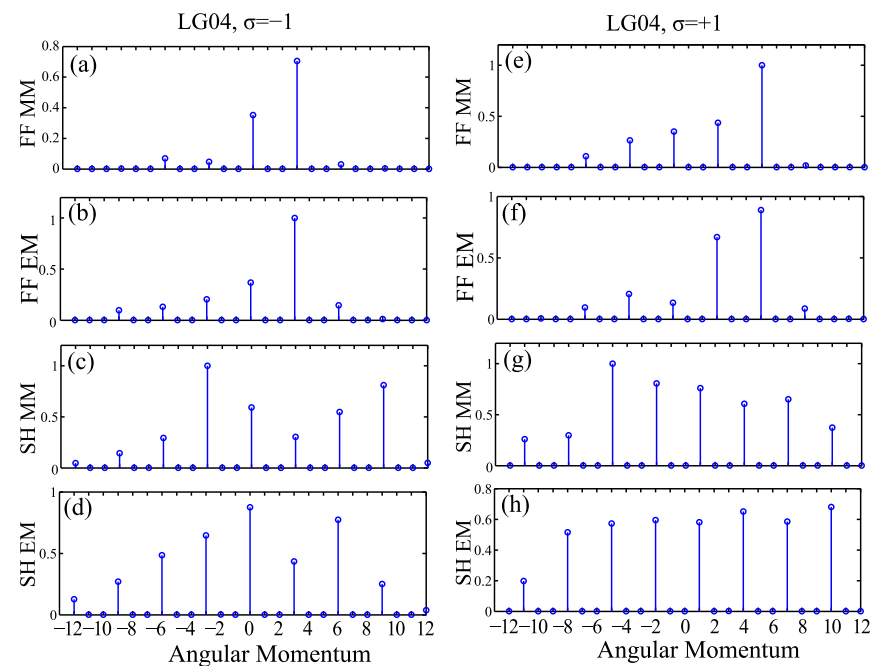

FIG. 9. Multipolar decompositions of the fundamental and SH scattered waves from the silicon C3-D chiral cluster in terms of angular momentum channels. MM and EM denote magnetic multipole and electric multipole, respectively. The silicon C3-D chiral cluster is illuminated by the RCP $\mathrm{LG}_{04}$ beam (left panels) and LCP LG $\mathrm{LG}_{04}$ beam (right panels). (a), (b), (e), and (f) correspond to scattered waves at FF, whereas (c), (d), (g), and (h) correspond to wave scattering at $\mathrm{SH}$.

LG beam with $l=4$ and $\sigma=-1$, the quantum numbers characterizing the total optical angular momentum are $j_{\text {sca }}=0, \pm 3, \pm 6, \pm 9, \ldots$, for both the scattered fundamental and $\mathrm{SH}$ fields. For the incident $\mathrm{LG}$ beam with $l=$ 4 and $\sigma=+1$, the scattered fundamental field has the quantum numbers $j_{\text {sca }}=\ldots,-7,-4,-1,2,5,8, \ldots$, whereas the scattered $\mathrm{SH}$ field has the quantum numbers $j_{\text {sca }}=$ $\ldots,-11,-8,-5,-2,1,4,7,10, \ldots$ These predictions, based on Eq. (2), completely agree with the multipole decomposition weights of the total angular momentum of the scattered fundamental and $\mathrm{SH}$ fields presented in Fig. 9.

The scattering of circularly polarized $\mathrm{LG}_{04}$ beams from plasmonic chiral clusters is also investigated. The plasmonic chiral cluster has the same configuration as the silicon one and is made of gold. The radius of each gold nanosphere is chosen to be $100 \mathrm{~nm}$ so that the cluster as a whole has strong surface plasmon resonances in the optical frequency region. The SH conversion efficiencies of the plasmonic (gold) and dielectric (silicon) chiral clusters illuminated by LCP and RCP $\mathrm{LG}_{04}$ beams are compared in Fig. 10. Note that the conversion efficiency of the dielectric chiral cluster is normalized to the cross-section area of the plasmonic and dielectric nanosphere, respectively, so that it gives us a per-particle quantification of the nonlinear conversion efficiency. The power of the incident LG beam for the dielectric and plasmonic nanostructures is set to be same, $P_{\text {in }}=2 \mathrm{~W}$. As we can see in Fig. 10, the dielectric chiral cluster made of high-permittivity silicon nanospheres supports both electric and magnetic resonances, leading to similar conversion efficiency when compared to that of the plasmonic chiral cluster. Considering that the much lower optical losses in dielectrics allow them to sustain higher optical power, it is possible that the dielectric nanostructures can provide orders of magnitude higher SH conversion efficiency, as compared to their plasmonic counterparts.
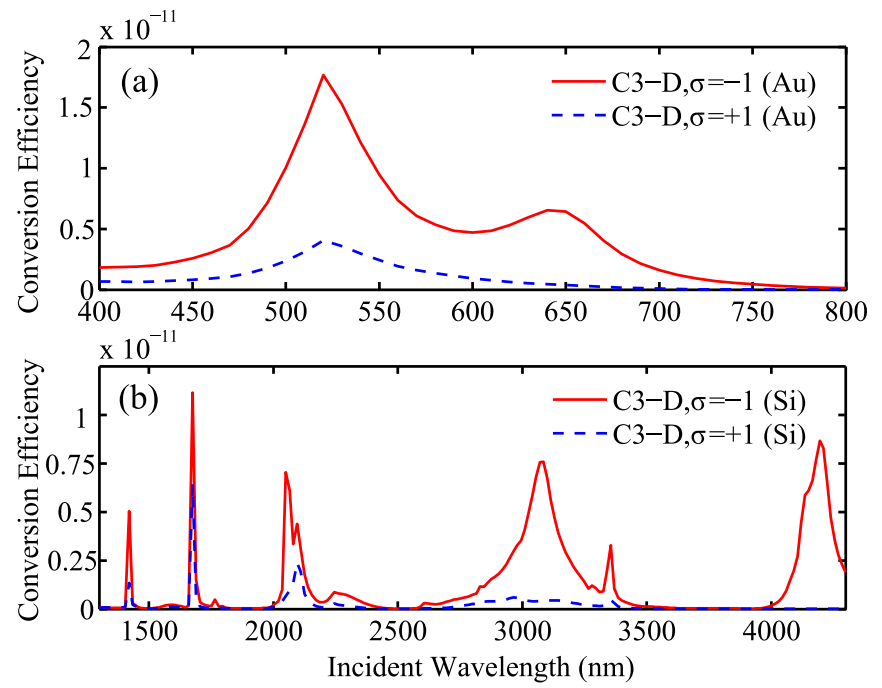

FIG. 10. SH conversion efficiencies of C3-D chiral clusters illuminated by LCP and RCP LG $_{04}$ beams. (a) Plasmonic (gold) chiral cluster. (b) Dielectric (silicon) chiral cluster.

\section{CONCLUSION}

In summary, we have studied the scattering of circularly polarized Laguerre-Gaussian beams from single plasmonic and dielectric nanospheres made of centrosymmetric materials, as well as chiral clusters made of such nanospheres, by means of the boundary element method. The phase singularities and vorticities in the longitudinal components of the fundamental and second-harmonic fields have been discussed and their relationship with the rotational symmetry of the scatterer has been analyzed. The doubling of the angular momentum and frequency during the second-harmonic nonlinear spin-orbit-interaction process has been demonstrated. The resonant excitation of multipolar optical modes in the dielectric nanosphere, leading to significant enhancement of scattered radiation at second harmonic, has been analyzed in detail. A general angular momenta conservation law, including the quasi-angular-momentum of chiral nanostructures with $N$-fold rotational symmetry, has been formulated to describe how the rotational symmetry properties affect the transfer of optical angular momentum from incident beams to both the scattered fundamental and second-harmonic fields. Our study reveals that, as compared to plasmonic nanostructures, dielectric nanostructures with pronounced resonance peaks and high ablation optical power threshold are better candidates for applications in near-field optical microscopy, nonlinear biosensing, optical angular momentum multiplexing/demultiplexing, and quantum information coding and decoding.

\section{ACKNOWLEDGMENTS}

This work was supported by the Research Grants Council of Hong Kong (GRF 716713, GRF 17207114, and GRF 17210815), National Science Foundation of China (No. 61271158 and No. 61201122), Hong Kong ITP/045/14LP, Hong Kong UGC AoE/-04/08, the Collaborative Research Fund (No. C7045-14E) from the Research Grants Council of Hong Kong, and Grant CAS14601 from the CAS-Croucher 
Funding Scheme for Joint Laboratories. This project is supported in part by a Hong Kong UGC Special Equipment Grant (No. SEG HKU09). N.C.P. acknowledges support from the European Research Council, Grant Agreement No. ERC2014-CoG-648328. X.Y.Z.X. thanks Prof. W. C. H. Choy and Prof. W. C. Chew for constructive discussions and suggestions.
[1] H. He, M. E. J. Friese, N. R. Heckenberg, and H. RubinszteinDunlop, Direct Observation of Transfer of Angular Momentum to Absorptive Particles from a Laser Beam with a Phase Singularity, Phys. Rev. Lett. 75, 826 (1995).

[2] S. H. Simpson and S. Hanna, Rotation of absorbing spheres in Laguerre-Gaussian beams, J. Opt. Soc. Am. A 26, 173 (2009).

[3] M. Padgett and R. Bowman, Tweezers with a twist, Nat. Photon. 5, 343 (2011).

[4] J. Wang, J.-Y. Yang, I. M. Fazal, N. Ahmed, Y. Yan, H. Huang, Y. Ren, Y. Yue, S. Dolinar, M. Tur, and A. E. Willner, Terabit freespace data transmission employing orbital angular momentum multiplexing, Nat. Photon. 6, 488 (2012).

[5] N. Bozinovic, Y. Yue, Y. Ren, M. Tur, P. Kristensen, H. Huang, A. E. Willner, and S. Ramachandran, Terabit scale orbital angular momentum mode division multiplexing in fibers, Science 340, 1545 (2013).

[6] M. Mirhosseini, O. S. Magana-Loaiza, M. N. O’Sullivan, B. Rodenburg, M. Malik, D. J. Gauthier, and R. W. Boyd, Highdimensional quantum cryptography with twisted light, New J. Phys. 17, 033033 (2015).

[7] A. Mair, A. Vaziri, G. Weihs, and A. Zeilinger, Entanglement of the orbital angular momentum states of photons, Nature (London) 412, 313 (2001).

[8] J. W. R. Tabosa and D. V. Petrov, Optical Pumping of Orbital Angular Momentum of Light in Cold Cesium Atoms, Phys. Rev. Lett. 83, 4967 (1999).

[9] S. Chen, F. Zeuner, M. Weismann, B. Reineke, G. Li, V. K. Valev, K. W. Cheah, N. C. Panoiu, T. Zentgraf, and S. Zhang, Giant nonlinear optical activity of achiral origin in planar metasurfaces with quadratic and cubic nonlinearities, Adv. Mater. 28, 2992 (2016).

[10] G. Li, S. Chen, N. Pholchai, B. Reineke, P. W. H. Wong, E. Y. B. Pun, K. W. Cheah, T. Zentgraf, and S. Zhang, Continuous control of the nonlinearity phase for harmonic generations, Nat. Mater. 14, 607 (2015).

[11] K. Dholakia, N. B. Simpson, M. J. Padgett, and L. Allen, Secondharmonic generation and the orbital angular momentum of light, Phys. Rev. A 54, R3742 (1996).

[12] J. Courtial, K. Dholakia, L. Allen, and M. J. Padgett, Secondharmonic generation and the conservation of orbital angular momentum with high-order Laguerre-Gaussian modes, Phys. Rev. A 56, 4193 (1997).

[13] S. M. Li, L. J. Kong, Z. C. Ren, Y. Li, C. Tu, and H. T. Wang, Managing orbital angular momentum in secondharmonic generation, Phys. Rev. A 88, 035801 (2013).

[14] N. V. Bloch, K. Shemer, A. Shapira, R. Shiloh, I. Juwiler, and A. Arie, Twisting Light by Nonlinear Photonic Crystals, Phys. Rev. Lett. 108, 233902 (2012).

[15] W. T. Buono, L. F. C. Moraes, J. A. O. Huguenin, C. E. R. Souza and A. Z. Khoury, Arbitrary orbital angular momentum addition in second harmonic generation, New J. Phys. 16, 093041 (2014).

[16] J. E. Sipe, V. C. Y. So, M. Fukui, and G. I. Stegeman, Analysis of second-harmonic generation at metal surfaces, Phys. Rev. B 21, 4389 (1980).
[17] L. Cao, N. C. Panoiu, and R. M. Osgood, Surface secondharmonic generation from surface plasmon waves scattered by metallic nanostructures, Phys. Rev. B 75, 205401 (2007).

[18] C. G. Biris and N. C. Panoiu, Second harmonic generation in metamaterials based on homogeneous centrosymmetric nanowires, Phys. Rev. B 81, 195102 (2010).

[19] C. Ciraci, E. Poutrina, M. Scalora, and D. R. Smith, Origin of second-harmonic generation enhancement in optical split-ring resonators, Phys. Rev. B 85, 201403 (2012).

[20] C. G. Biris and N. C. Panoiu, Nonlinear Surface-Plasmon Whispering-Gallery Modes in Metallic Nanowire Cavities, Phys. Rev. Lett. 111, 203903 (2013).

[21] R. Czaplicki, J. Makitalo, R. Siikanen, H. Husu, J. Lehtolahti, M. Kuittinen, and M. Kauranen, Second-harmonic generation from metal nanoparticles: Resonance enhancement versus particle geometry, Nano Lett. 15, 530 (2015).

[22] J. Butet, K. Thyagarajan, and O. J. F. Martin, Ultrasensitive optical shape characterization of gold nanoantennas using second harmonic generation, Nano Lett. 13, 1787 (2013).

[23] V. K. Valev, Characterization of nanostructured plasmonic surfaces with second harmonic generation, Langmuir 28, 15454 (2012).

[24] G. Bautista, M. J. Huttunen, J. Makitalo, J. M. Kontio, J. Simonen, and M. Kauranen, Second-harmonic generation imaging of metal nano-objects with cylindrical vector beams, Nano Lett. 12, 3207 (2012).

[25] C. G. Biris and N. C. Panoiu, Excitation of dark plasmonic cavity modes via nonlinearly induced dipoles: Applications to near-infrared plasmonic sensing, Nanotechnol. 22, 235502 (2011).

[26] M. Kauranen and A. V. Zayats, Nonlinear plasmonics, Nat. Photon. 6, 737 (2012).

[27] M. R. Shcherbakov, D. N. Neshev, B. Hopkins et al., Enhanced third-harmonic generation in silicon nanoparticles driven by magnetic response, Nano Lett. 14, 6488 (2014).

[28] L. Carletti, A. Locatelli, O. Stepanenko, G. Leo, and C. De Angelis, Enhanced second-harmonic generation from magnetic resonance in AlGaAs nanoantennas, Opt. Express 23, 26544 (2015).

[29] A. Al-Jarro, C. G. Biris, and N. C. Panoiu, Resonant mixing of optical orbital and spin angular momentum by using chiral silicon nanosphere clusters, Opt. Express 24, 6945 (2016).

[30] L. Allen, M. W. Beijersbergen, R. J. C. Spreeuw, and J. P. Woerdman, Orbital angular momentum of light and the transformation of Laguerre-Gaussian laser modes, Phys. Rev. A 45, 8185 (1992).

[31] D. L. Andrews, Structured Light and Its Applications: An Introduction to Phase-Structured Beams and Nanoscale Optical Forces (Academic Press-Elsevier, Burlington, 2008).

[32] X. Y. Z. Xiong, L. J. Jiang, W. E. I. Sha, Y. H. Lo, M Fang, W. C. Chew, and W. C. H. Choy, Strongly enhanced and directionally tunable second-harmonic radiation by a plasmonic particle-in-cavity nanoantenna, Phys. Rev. A 94, 053825 (2016). 
[33] Y. Zhang, N. K. Grady, C. Ayala-Orozco, and N. J. Halas, Three-dimensional nanostructures as highly efficient generators of second harmonic light, Nano Lett. 11, 5519 (2011).

[34] J. Butet, P.-F. Brevet, and O. J. F. Martin, Optical second harmonic generation in plasmonic nanostructures: From fundamental principles to advanced applications, ACS Nano 9, 10545 (2015).

[35] J. I. Dadap, J. Shan, K. B. Eisenthal, and T. F. Heinz, SecondHarmonic Rayleigh Scattering from a Sphere of Centrosymmetric Material, Phys. Rev. Lett. 83, 4045 (1999).

[36] T. F. Heinz, Second-order nonlinear optical effects at surfaces and interfaces, in Nonlinear Surface Electromagnetic Phenomena, edited by H. E. Ponath and G. I. Stegeman (Elsevier, Amsterdam, 1991), p. 353.

[37] D. Krause, C. W. Teplin, and C. T. Rogers, Optical surface second harmonic measurements of isotropic thin-film metals: Gold, silver, copper, aluminum, and tantalum, J. Appl. Phys. 96, 3626 (2004).

[38] M. Falasconi, L. C. Andreani, A. M. Malvezzi, M. Patrini, V. Mulloni, and L. Pavesi, Bulk and surface contributions to second-order susceptibility in crystalline and porous silicon by second-harmonic generation, Surf. Sci. 481, 105 (2001).

[39] P. B. Johnson and R. W. Christy, Optical constants of noble metals, Phys. Rev. B 6, 4370 (1972).

[40] B. Tatian, Fitting refractive-index data with the Sellmeier dispersion formula, Appl. Opt. 23, 4477 (1984).

[41] M. D. Williams, M. M. Coles, K. Saadi, D. S. Bradshaw, and D. L. Andrews, Optical Vortex Generation from Molecular Chromophore Arrays, Phys. Rev. Lett. 111, 153603 (2013).

[42] M. M. Coles, M. D. Williams, K. Saadi, D. S. Bradshaw, and D. L. Andrews, Chiral nanoemitter array: A launchpad for optical vortices, Laser Photon. Rev. 7, 1088 (2013).

[43] K. Sakai, K. Nomura, T. Yamamoto, and K. Sasaki, Excitation of multipole plasmons by optical vortex beams, Sci. Rep. 5, 8431 (2015).

[44] C. Menzel, C. Rockstuhl, T. Paul, and F. Lederer, Retrieving effective parameters for quasiplanar chiral metamaterials, Appl. Phys. Lett. 93, 233106 (2016).

[45] J. D. Jackson, Classical Electrodynamics, 3rd ed. (John Wiley \& Sons, New York, 2007). 